\title{
PATRONES DE INTERCAMBIO CONDUCTUAL QUE PREDICEN EL ÉXITO EN COMBATES DE TAEKWONDO
}

\author{
Ana del Refugio Cervantes Herrera \\ Francisco Javier Pedroza Cabrera \\ Universidad Autónoma de Ciudad Juárez \\ ana.cervantesherrera@gmail.com \\ https://doi.org/10.17060/ijodaep.2017.n1.v2.947
}

Fecha de Recepción: 3 Marzo 2017

Fecha de Admisión: 1 Abril 2017

\section{RESUMEN}

Si bien el comportamiento deportivo se estudia frecuentemente, el hecho de que sea a través de instrumentos indirectos, lleva a que en muchos casos se desconozcan las variables de las cuales depende la ejecución exitosa del deportista. Atendiendo a esto, el presente trabajo tuvo como objetivo ubicar a través de la observación directa los patrones de interacción que predicen la victoria en combates de Taekwondo. Se video-grabaron 54 contiendas de deportistas cinta negra (de 13 a 19 años), en torneos oficiales. Las contiendas fueron analizadas a través del Sistema de Observación Directa de la Ejecución del Deportista en Combate con el software Observer XT y posteriormente analizadas con el software THEME- $V$, con la finalidad de encontrar patrones ocultos en los intercambios sociales y con el paquete estadístico SPSS. Se encontraron diferencias estadísticamente significativas en el tipo y orden de las técnicas utilizadas por los que ganaron y perdieron en las diferentes contiendas observadas. También se ubicaron diferencias en los patrones conductuales que involucran a ambos contendientes. Se discuten las implicaciones de los resultados en el diseño de estrategias de entrenamiento.

Palabras Clave: Patrones conductuales, Taekwondo, Observación, Entrenamiento.

\section{ABSTRACT}

Behavioral patterns of exchange that predicts success in taekwondo combats

The sporting behavior is traditionally studied through indirect instruments, nevertheless the results obtained from observational methodology allows locating the variables on which depends the successful execution of the athlete. In response to this, the present study aimed to locate the interaction patterns that predict victory in Taekwondo fights. 54 fights were videotaped, and were analyzed through a behavioral catalog. Statistically significant differences were found in the tech- 


\section{PATRONES DE INTERCAMBIO CONDUCTUAL QUE PREDICEN EL ÉXITO EN COMBATES DE TAEKWONDO}

nique used by those whom won and whom lost strife and the use of tactical resources. The implications of the results in success in combat and training design are discussed.

Key words: Taekwondo, Behavioral patterns, Observation, Training.

\section{INTRODUCCIÓN}

Tradicionalmente el comportamiento deportivo se ha investigado ya sea en términos de variables fisiológicas (Savage \& Stuart, 2016), mecánicas (Estevan, Jandacka \& Falco, 2013), o perceptuales, a través de instrumentos indirectos tales como auto-reportes e instrumentos psicométricos (Barkoukis, Lazuras \& Tsorbatzoudis, 2014), los cuales debido a su naturaleza proporcionan medidas globales y en las que datos finos como la frecuencia, duración, topografía y secuencia de la conducta quedan fuera (López \& Santoyo, 2004).

Paradójicamente, la tarea central para el psicólogo conductual en el ámbito deportivo es la identificación de las variables observables y cuantificables, de las cuales depende la ejecución exitosa del deportista (Cruz, 1991), ya que, para explicar los éxitos y fracasos de los deportistas es necesario analizar y comprender las diferentes morfologías de conducta que conforman las actividades involucradas en este comportamiento (Ardá \& Anguera, 2000). Esto se ha logrado lo los deportes de conjunto, en la revisión de secuencias ofensivas y sus resultados (Lapresa, Arana, Anguera, \& Garzón, 2013); sin embargo, ha quedado desatendida en los deportes de combate donde es posible encontrar un menor número de ejemplos.

En el estudio de los deportes de combate es importante tomar en cuenta su reglamentación y características para su adecuado estudio ya que, para un análisis completo de un deporte, se debe atender a los componentes que influirán en el resultado final de la competencia (Gutiérrez-Santiago et al., 2011); justamente estas condiciones son las que delimitan el tipo de interacciones sociales que pueden desarrollarse durante la justa en cada deporte en particular.

En los deportes de contacto (combate), los competidores interactúan tratando de impactar uno sobre el otro (marcar puntos), evitando ser impactados o realizar acciones que deriven en sanciones y por lo tanto en la pérdida de puntos (Gasset \& Iglesias, 2010; Iglesias, Gasset, González \& Anguera, 2010).

Específicamente en el taekwondo, el área de combate se restringe a un espacio de 8x8 mts., delimitada por un área de al menos dos metros. En cuanto a la variable tiempo, la contienda se encuentra delimitada por tres periodos de tres minutos y, en caso necesario, un tiempo extra (Fernández, 2004). Basado en la estructura funcional de las conductas posibles, éstas se han dividido en conductas ofensivas, defensivas y recursos tácticos (González, Iglesias, Anton \& Esparza, 2011) así como conductas antideportivas.

En este deporte, al igual que en el resto de los deportes de combate, los intercambios (interacciones) que suceden durante los episodios deportivos derivan en patrones de interacción entre competidores. Aun cuando la interacción que se genera en una actividad de combate es determinante para la consecución del triunfo, pocos trabajos se han enfocado en analizar dicha interacción. Uno de los equipos que se ha ocupado de la interacción en combate es el conformado por Iglesias y sus colaboradores, quienes en 2010 analizaron la influencia de la presión ambiental en la densidad y efectividad de las acciones en situaciones competitivas en este tipo de deportes (esgrima y taekwondo), encontrando patrones secuenciales de acciones de combate. Sin embargo, dicho trabajo deja fuera las secuencias tanto de ataque y defensa de los competidores, así como los patrones de intercambio derivados de estas.

Una manera efectiva para el estudio de los patrones de interacción de los combatientes en taekwondo es la metodología observacional. Ésta permite un estudio continuo en el tiempo, atendiendo 
a la variabilidad permanente que se produce en el comportamiento deportivo (Anguera \& Blanco, 2003). Así como, cuantificar momento a momento las interacciones ocurridas obteniendo patrones secuenciales recurrentes (Magnusson, 2000).

Tomando en cuenta esta información, el presente trabajo tiene el propósito de ubicar los patrones conductuales en combate de deportistas de taekwondo que predicen el éxito en diferentes grupos de edad.

\section{MÉTODO}

\section{Participantes}

84 deportistas que practican Taekwondo (ambos sexos) con cinturón negro. 54 de ellos pertenecen a la selección del estado de Aguascalientes y se distribuyen por categoría dede la siguiente manera: Sub13 $n=11$, Sub15 n=12, Sub17 $n=15$ y Sub20 $n=16$.

\section{Escenario}

Gimnasio olímpico de la ciudad de Aguascalientes; espacio que cuenta con un área de combate de $8 \times 8$ mts., y un área de seguridad de $2 \mathrm{mts}$.

\section{Instrumentos}

Sistema de Observación Directa de la Ejecución del Deportista en Combate (Pedroza \& Cervantes, en prensa). Instrumento que consta de 52 conductas, que abarcan los movimientos ofensivos, defensivos, uso de conductas antideportivas, así como las variables temporales, espaciales y el logro del punto. Se obtuvo un coeficiente Kappa superior a 0.84 en pares de observadores entrenados.

\section{Procedimiento}

Una vez obtenido el consentimiento informado, se llevó a cabo la videograbación de combates realizados durante tres torneos oficiales (olimpiadas regionales y nacionales) en los que participaron deportistas con cinta negra en Taekwondo, obteniéndose un total de 54 contiendas registrables.

Posteriormente se realizó el registro de observación de los videos con el Sistema de Observación Directa de la Ejecución del Deportista en Combate a través del software observacional OBSERVER XT®.

A partir de los datos arrojados por el registro de observación directa de los combates, se llevaron a cabo análisis de frecuencia de uso de las diferentes técnicas de ataque y defensa tanto en la totalidad de la muestra como al interior de las diferentes categorías de combate (sub13, sub15, sub17 y sub20) en el paquete estadístico SPSS-21. También se ha realizado la búsqueda de patrones ocultos a través del software de análisis THEME®.

\section{RESULTADOS}

Como resultado de las videograbaciones registro de combates y análisis paramétrico de datos, se encontraron diferencias significativas $(p<=0.05)$ en el uso de guardias; donde los participantes que ganaron la contienda incurrieron una mayor cantidad de tiempo en el uso de Guardia Derecha sin Brazo con una media de 92.23seg ( $D E=82.30)$, mientras que los que perdieron acumularon una media de $80.41 \mathrm{seg}(\mathrm{DE}=54.71)$. La situación inversa se presentó en el caso del uso de la Guardia Izquierda sin Brazo, la cual fue ejecutada por los que ganaron (GN) en promedio 35.30seg $(\mathrm{DE}=40.17)$, mientras que los que perdieron (PN) la realizaron con una media de $55.36 \mathrm{seg}$ $(\mathrm{DE}=59.90)$. 
En cuanto a las conductas de ataque, se presentaron diferencias significativas en la Patada Circular Derecha Trasera (PCDTRA) presentada con mayor frecuencia por los que perdieron la contienda con una tasa media de 2.31ejecuciones por minuto ( $D E=1.72)$, mientras que los GN la ejecutaron 1.78 ocasiones por minuto ( $D E=1.38$ ). También se encontraron diferencias significativas en el uso de Golpe con Puño Derecho (GOD); esta técnica se presentó con mayor frecuencia en el grupo de $G N(X=0.29, D E=0.53)$, que en el grupo que PN la pelea $(X=0.18, D E=0.35)$.

Otro grupo de conductas en las cuales se encontraron diferencias significativas es en cuatro tipos de obtención de puntos. En la obtención de un punto (PTU), se encuentra una mayor incidencia para el grupo que ganó la contienda $(X=9.33, D E=5.46)$, con respecto a los que perdieron $(X=3.97, D E=3.57)$. Una situación similar se presenta en el caso de la frecuencia de obtención de tres puntos, presentándose con mayor frecuencia para los $G N(X=1.13, D E=1.42)$, que para los PN $(X=0.36, D E=0.74)$. Lo mismo sucede en lo que se refiera a la obtención de cuatro puntos; en este caso los GN se presentó con una media de 0.21 ( $D E=0.57$ ), y en los PN con una media de 0.08 $(D E=0.35)$. Lo inverso se puede observar en cuanto a la obtención de punto por amonestación al contrincante (PPA), la cual benefició en mayor frecuencia a los $P N(X=1, D E=1.15)$, que a los $G N$ $(X=0.54, D E=0.79)$.

Por otra parte, en lo concerniente a la pérdida de un punto como consecuencia de la emisión de protesta por parte del oponente, que se presentó con una tasa mayor para los GN ( $X=0.10$, $\mathrm{DE}=0.31)$, que en los $\mathrm{PN}(\mathrm{X}=0.08, \mathrm{DE}=0.27)$.

También se llevó a cabo un análisis de medias a través de una Prueba t para muestras independientes con la finalidad de encontrar las conductas con diferencias estadísticamente significativas entre los dos tipos de participantes (GN y PN)para cada categoría de peso (sub13, sub15, sub27, y sub20); a continuación, se presentan los principales resultados por categorías de combate:

En lo concerniente a la categoría sub13 se recabaron un total de 13 contiendas; durante el análisis se comparó la conducta de los GN vs la de los PN encontrando diferencias significativas en la duración tres de las conductas defensivas (GDB, GISB y BPD), también en dos de los recursos tácticos (DPD) y FN), la conducta antideportiva CadeGnp, así como 13 conductas de ataque (ver tabla1). 
Tabla 1.- Conductas con diferencias entre GN y PN Sub13

\begin{tabular}{|l|l|l|l|l|}
\hline Conducta & F & gl & X GN & X PN \\
\hline Guardia Derecha con Brazo (GDB) & 7.31 & 12 & 106.02 & $74.79^{*}$ \\
\hline Guardia Izquierda sin Brazo (GISB) & 5.99 & 12 & 45.82 & $32.98^{*}$ \\
\hline Bloqueo Pie Derecho (BPD) & 6.30 & 12 & 0.06 & $0.23^{*}$ \\
\hline Distancia Pie Derecho (DPD) & 8.45 & 12 & 0.06 & $0.50^{*}$ \\
\hline Finta (FN) & 5.66 & 12 & 4.99 & $4.72^{*}$ \\
\hline Cade Golpes no Permitidos (CadeGnp) & 14.45 & 12 & 0.29 & $0.45^{*}$ \\
\hline Patada Frente Izquierda Trasera & 5.76 & 12 & 0.00 & $0.06^{*}$ \\
\hline Patada Frente Derecha Delantera & 22.25 & 12 & 0.00 & $0.09^{* *}$ \\
\hline Patada Circular Salto Trasera & 5.50 & 12 & 0.14 & $0.02^{*}$ \\
\hline Patada Lado Izquierda Salto Delantera & 5.76 & 12 & 0.04 & $0.00^{*}$ \\
\hline Patada Lado Derecha Salto Delantera & 5.76 & 12 & 0 & $0.02^{*}$ \\
\hline Patada Descendente Izquierda Delantera & 5.76 & 12 & 0.02 & $0.00^{*}$ \\
\hline Patada Descendente Izquierda Trasera & 10.71 & 12 & 0.02 & $0.10^{*}$ \\
\hline Patada Descendente Derecha Delantera & 10.36 & 12 & 0.02 & $0.11^{*}$ \\
\hline Patada Descendente Derecha Salto Trasera & 5.76 & 12 & 0.00 & $0.02^{*}$ \\
\hline Patada Gancho Derecha & 23.61 & 12 & 0.00 & $0.07^{* *}$ \\
\hline Patada Doble Círculo Derecha Trasera & 5.76 & 12 & 0.05 & $0.00^{*}$ \\
\hline
\end{tabular}

${ }^{*} \mathrm{p}<0.05,{ }^{* *} \mathrm{p}<0.001$

En lo que respecta la categoría sub15 se analizaron un total de 13 combates comparando las conductas de los GN vs los PN, se encontraron diferencias estadísticamente significativas en el uso de un tipo de conducta defensiva (BBD), el uso de la conducta táctica de uso de DPD, en ocho conductas de ataque (incluyendo el uso de Golpe con puño y seis tipos de patadas), así como en la 
obtención de puntos, uso de Conducta Antideportiva, obtención de amonestaciones y pérdida de puntos por acumulación de amonestación (ver tabla 2).

Tabla 2.- Conductas con diferencias entre GN y PN Sub15

\begin{tabular}{|c|c|c|c|c|c|}
\hline Conducta & $\mathbf{F}$ & $P$ & gl & $\mathbf{X}$ GN & X PN \\
\hline Bloqueo con Brazo Derecho (BBD) & 5.730 & .038 & 10 & 0.81 & 0.48 \\
\hline Distancia Pie Derecho (DPD) & 6.250 & .031 & 10 & 0.00 & 0.02 \\
\hline Golpe puño Izquierdo & 6.250 & .031 & 10 & 0.02 & 0.00 \\
\hline Patada Frente Derecha Trasera & 6.250 & .031 & 10 & 0.02 & 0.00 \\
\hline $\begin{array}{|lll|}\text { Patada Circular Izquierda Salto } \\
\text { Delantera }\end{array}$ & 6.607 & .028 & 10 & 0.08 & 0.60 \\
\hline Patada Lado Izquierda Delantera & 22.891 & .001 & 10 & 0.00 & 0.06 \\
\hline $\begin{array}{|lll|}\text { Patada } & \text { Descendente } & \text { Izquierda } \\
\text { Delantera } & & \\
\end{array}$ & 32.529 & .000 & 10 & 0.00 & 0.04 \\
\hline Patada Giro Recto Izquierda Trasera & 6.250 & .031 & 10 & 0.02 & 0.00 \\
\hline Patada con Obtención de un Punto & 6.036 & .034 & 10 & 9.50 & 2.00 \\
\hline $\begin{array}{|llll|}\text { Patada con Obtención de Tres } \\
\text { Puntos } & & & \\
\end{array}$ & 8.929 & .014 & 10 & 1.17 & 0.17 \\
\hline Punto por Amonestación & 19.620 & .001 & 10 & 0.17 & 1.00 \\
\hline Cade Amonestación & 15.079 & .003 & 10 & 0.24 & 0.06 \\
\hline $\begin{array}{l}\text { Pérdida de un punto por } \\
\text { amonestación }\end{array}$ & 6.250 & .031 & 10 & 0.00 & 0.17 \\
\hline
\end{tabular}

En la categoría sub17 se analizaron en total 13 combates, a partir de los cuales se encontraron diferencias significativas entre GN y PN en 29 conductas que abarcan el uso del espacio (Zona), Conductas defensivas, conductas de ataque, la emisión de conducta Antideportiva, la pérdida de puntos y la obtención de Puntos por Amonestaciones al oponente (ver tabla 3). 
Tabla 3.- Conductas con diferencias entre GN y PN Sub17

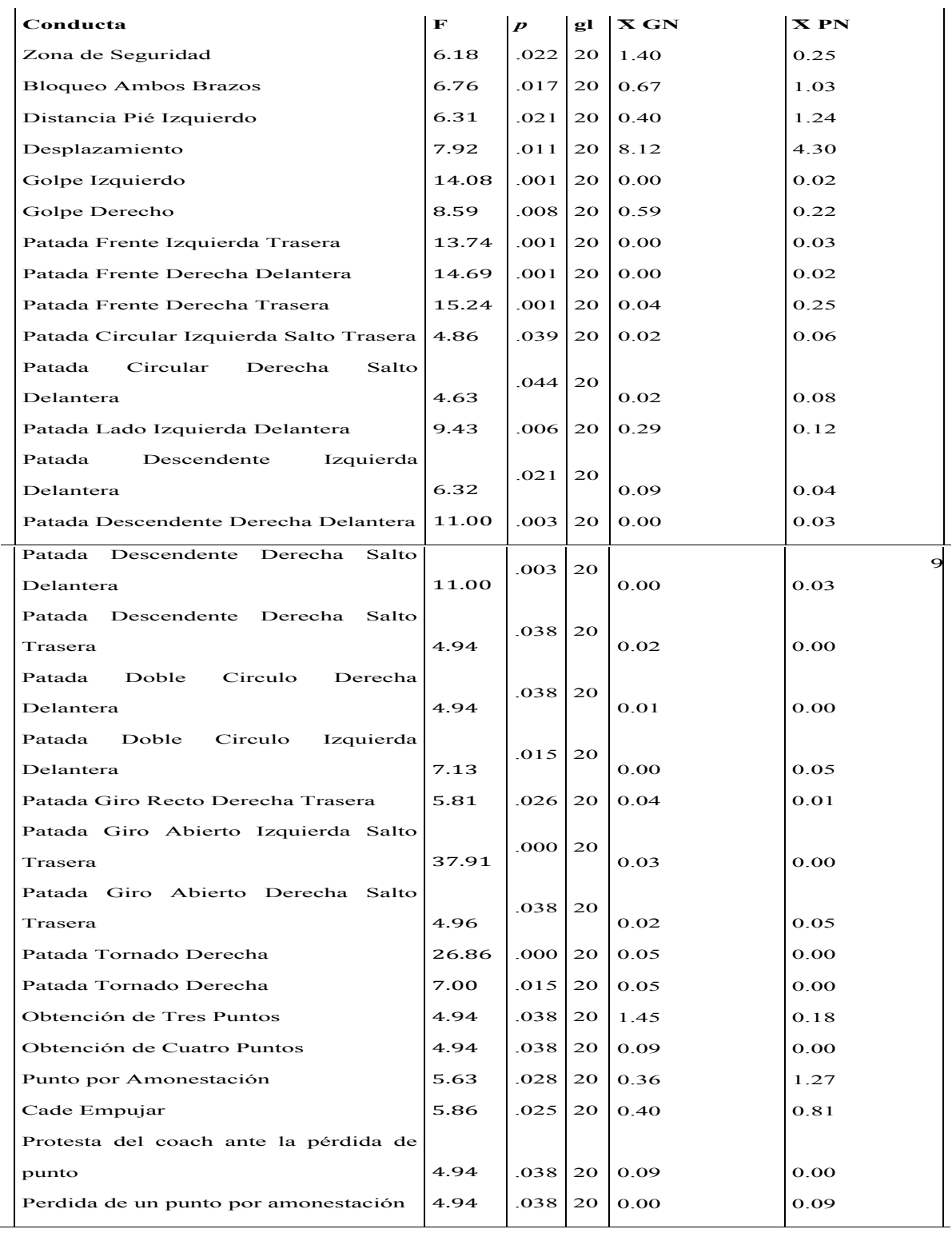

En la categoría sub20 se trabajó con 15 combates (ver tabla 5) encontrándose diferencias entre Ios GN y los PN en un total de 21 conductas que incluyen una conducta defensiva, 16 tipos de patadas, obtención de puntos y la emisión de CADE, así como la pérdida de puntos derivada de la reincidencia en CADE (tabla 4). 
Tabla 4.- Conductas con diferencias entre GN y PN Sub 20

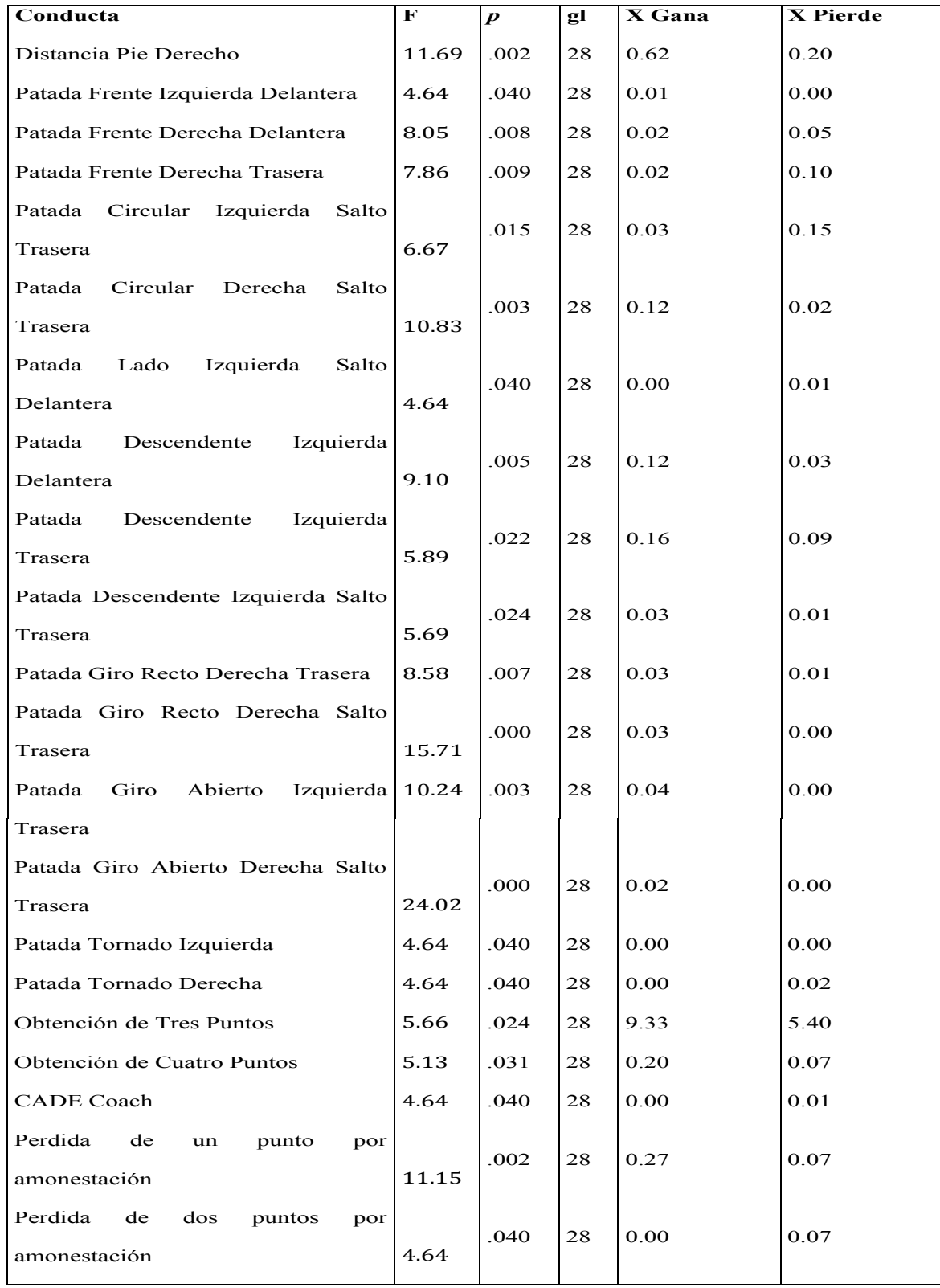


Se realizó posteriormente un análisis en el software THEME en busca de patrones ocultos en el comportamiento de los deportistas en combate incluyendo la totalidad de peleas registradas, ubicándose un total de 24 patrones ocultos; debido a la densidad de datos en el presente documento se presentan solamente tres de ellos (Figura 1).

Figura 1.- Presenta tres patrones (uno de uso de recuso táctico y 2 de combate y defensa).

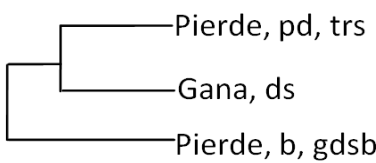

A

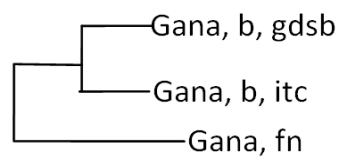

B

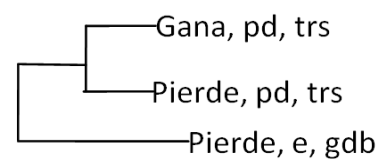

C

En el primero de ellos (panel A de la figura 2) se muestra un patrón que comienza con una conducta de ataque por parte del participante PN (patada con pierna derecha, trasera) [PD,TRS] que deriva en la perdida de guardia (GDSB), a la cual el participante GN responde con un desplazamiento (DS). El segundo patrón presentado en la figura 2 (panel B) presenta la pérdida de guardia por parte del deportista que gana la pelea, seguida una conducta antideportiva (interrupción del combate [ITC]), el uso de un recurso táctico (FN). En el panel C es posible ver un patrón de ataque en espejo iniciado por el participante que gana la contienda, en el que el que la pierde logra mantener la guardia.

Con la finalidad de conocer la influencia del paso del tiempo en la conducta del deportista, se realizó un análisis en busca de las secuencias de ataque que derivaron en punto en cada uno de los rounds. En lo referente al primer round, se encontraron 15 patrones diferentes que derivaron en la obtención de uno 0 más puntos. Estos patrones se presentaron con una frecuencia que fue de 1 hasta 54 ocasiones; a continuación se presentan los más frecuentes.

Los patrones ubicados se dividieron en tres tipos (los que inician con una técnica de ataque, los que comienzan con el uso de un recurso táctico y el tercero defensa y conducta antideportiva. En la tabla 5 se presentan los patrones encontrados que se presentaron con una frecuencia $>=10$; indicando el tipo de participante (el que obtiene el punto [SP] y el que no lo obtiene [NP]), ligado al tipo de conducta emitida por un guion y separados por una coma con la siguiente acción realizada en el patrón. 
Tabla 5.- Ocurrencia por round de cada tipo de patrón que deriva en punto

\begin{tabular}{|c|c|c|c|}
\hline ATAQUE & Round 1 & Round 2 & Round 3 \\
\hline SP-Ataque, SP-Punto & 51 & 35 & 46 \\
\hline SP-Patada, SP-Punto, NP-Bloqueo & $<10$ & 39 & $<10$ \\
\hline NP-Patada, SP-Patada, SP Punto & $<10$ & 27 & $<10$ \\
\hline SP-Patada, SP-Punto, NP-Táctico & $<10$ & 16 & 11 \\
\hline SP-Patada, SP-Punto, NP- Patada & & 10 & \\
\hline TÁCTICO & Round 1 & Round 2 & Round 3 \\
\hline NP-táctico, SP-Patada, SP-Punto & 18 & $<10$ & $<10$ \\
\hline NP-CambiaGuardia, SP-Patada, SP- Punto & 36 & 25 & 21 \\
\hline \multicolumn{4}{|l|}{ NP-CambiaGuardia, SP-Patada, SP- Punto, NP- } \\
\hline Bloqueo & 18 & 22 & 15 \\
\hline \multicolumn{4}{|l|}{ NP-CambiaGuardia, SP-Patada, SP- Punto, NP- } \\
\hline Táctico & $<10$ & 10 & $<10$ \\
\hline DEFENSA / ANTIDEPORTIVA & Round 1 & Round 2 & Round 3 \\
\hline NP-Bloqueo, SP-Patada, SP-Punto & 26 & $<10$ & $<10$ \\
\hline NP-AntiDeportiva, NP-Amonestación, SP-Punto & 12 & 23 & 25 \\
\hline SP-AntiDeportiva, SP-Patada, SP-Punto & $<10$ & 10 & $<10$ \\
\hline
\end{tabular}

\section{DISCUSIÓN}

Durante el desarrollo del presente trabajo se incluyeron conductas que inciden directamente en el marcador que no fueron incluidas en trabajos previos tales como la ejecución en conductas antideportivas (CADE) tanto por parte del competidor como por el coach, la pérdida de puntos derivada de la reincidencia en el uso de CADE y la obtención de puntos y pérdida de puntos (en las diferentes cantidades posibles para cada tipo de técnica).

En cuanto al uso de la conducta Desplazamiento por parte de los participantes que ganan la pelea, se presenta asociada a la emisión de conductas de ataque por parte del oponente. De manera que los deportistas que ganan la contienda hacen uso de esta técnica tal como se espera que lo hagan, lo que significa mayor efectividad cuando se trata de evitar que su contrincante marque puntos. 
Otra conducta que afecta la obtención (y pérdida de puntos) y por lo tanto el marcador final es la incidencia de Conducta Antideportiva tanto por parte del deportista como por parte del coach; estas conductas se presentaron con mayor frecuencia en el caso de los combatientes que pierden la pelea, en quienes también se presentó mayor ocurrencia de pérdida de puntos, así como adjudicaciones de punto al contrincante por amonestación al deportista. De manera que el uso de estas conductas puede derivar directamente en la pérdida de la pelea, ya que si bien el deportista que gana presenta mayor efectividad en sus ataques, la pérdida de puntos agrava la situación de los participantes que pierden disminuyendo aún más la probabilidad de obtener el triunfo. Tener a disposición esta información permitirá a los entrenadores diseñar programas que les permitan controlar algunas de las variables que afectan el desempeño deportivo (Cruz, 1991).

En cuanto al análisis de frecuencia de uso de conductas en las diferentes categorías de combate, se muestra que las conductas en las que se presentan diferencias significativas son distintas en los diferentes grupos de edad. Así, mientras en la categoría sub13 existe una mayor incidencia de uso de guardia y fintas por parte de los contrincantes que ganan, esto no se presenta en el resto de las categorías. Por otra parte, en tres de cuatro categorías (sub13, sub15 y sub17), el grupo de GN emitió una mayor variedad de conductas de ataque; no así en la categoría sub20, lo cual puede indicar que los participantes de este último grupo poseen una mayor efectividad en la elección de la conducta adecuada (probablemente debido a la mayor exposición a situaciones de combate). Cabe resaltar que los resultados del resto de las categorías indican que una mayor variabilidad conductual es predictor de victoria en combate, ya que dificulta la predicción de la conducta por parte su oponente. Por otra parte, si bien en todas las categorías ambos contrincantes emitieron alguna $\mathrm{CADE}$, Ios contrincantes que perdieron la pelea la emitieron con una frecuencia mayor en las categorías sub13 y sub20, mientras que su emisión derivó en una mayor pérdida de puntos (o suma para el contrincante) en el caso de las categorías sub15 y sub20, de manera que su uso tiende a disminuir el marcador a largo plazo.

Por su parte, los patrones arrojados por el THEME muestran un adecuado uso de la técnica Desplazamiento por parte de los participantes que ganaron la contienda; también hacen uso de interrupción del combate (ITC) e implementan el uso de estrategias tácticas (FN) cuando se encuentran sin guardia (GDSB), lo cual les impide a los contendientes atacar en ese momento. Por su parte, los que perdieron presentan conductas de ataque en espejo de sus contrincantes; esto pude fungir como indicador de que estos deportistas presentan dificultades para ubicar la técnica a implementar.

En lo concerniente a la influencia del tiempo en la conducta de los deportistas en términos de la frecuencia de uso de algún tipo de técnica en cada round; cabe resaltar que los patrones iniciados con un ataque por parte del participante que obtiene el punto se presentan con mayor frecuencia en el primer y último round, mientras que los iniciados para el participante que no lo obtiene se presentan más el segundo round, junto con los que inician con una conducta antideportiva por parte del que obtiene el punto; esto puede indicar una ventaja inicial en el marcador por parte del participante que logra marcar puntos, así como un aumento en la cantidad y disminución en la calidad de intentos de marcar punto por aquellos deportistas que pierden la pelea.

Además, la densidad relativamente estable de puntos conseguidos cuando el que obtiene el punto realiza un ataque ante un cambio de guardia por parte del que no lo obtiene, indica una mayor probabilidad de obtención de punto al atacar cuando una conducta de este tipo (cambio de guardia) se presenta.

Es posible concluir que una alta variabilidad conductual, el uso de recursos tácticos y de defensa, así como una baja densidad de emisión de CADE por parte del deportista así como de su Coach, 


\section{PATRONES DE INTERCAMBIO CONDUCTUAL QUE PREDICEN EL ÉXITO EN COMBATES DE TAEKWONDO}

predicen en conjunto el éxito en la justa deportiva. Finalmente, es importante resaltar que si bien hasta el momento se ha logrado recabar y analizar un volumen importante de datos, aún es necesario realizar análisis con una mayor cantidad de datos que tomen en cuenta la experiencia de los deportistas.

\section{REFERENCIAS}

Anguera, M. T. \& Blanco, A. (2003). Registro y codificación del comportamiento deportivo. En A. Hernández-Mendo (Ed.), Psicología del Deporte Vol. Il: Metodología (pp. 6-34). Buenos Aires: Tulio Guterman.

Barkouskis, V., Lazuras, L., \& Tsorbatzoudis, H. (2014). Beliefs about the causes of success in sports and susceptibility for doping use in adolescent athletes. Journal of sports sciences, 32(3), 212219.

Cruz, J. (1991). Historia de la Psicología del deporte. En J. Riera y J. Cruz (Eds.), Psicología del Deporte. Aplicaciones y perspectivas (pp. 13-43). Barcelona: Martínez Roca.

Estevan, I., Jandacka, D., \& Falco, C. (2013). Effect if stance position on kick performance in taekwondo. Journal of sports sciences, 31(16), 1815-1822. doi:10.1080/02640414.2013.803590

Fernández, R. (2004). Nueva metodología para la enseñanza de las técnicas de pateos en la práctica del taekwondo (WTF). Revista Digital, 10(75). Recuperado de http://www.efdeportes.com/

Gasset, A., \& Iglesias, X. (2010). Caracterización de la presión temporal como factor ambiental de la conducta humana en situación de conflicto. El caso de la esgrima de espada. Comunicación presentada al V Congreso Internacional de Educación Física, 131-137 [CD].

González, C. Iglesias, X., Anton, J. \& Esparza, G. (2011). Sistematización de la acción táctica en el taekwondo de alta competición. Apunts. Educación física y deportes, 103, 56-67.

Gutiérrez-Santiago, A., Prieto, I., Camerino, 0. \& Anguera, T. (2011) The temporal structure of judo bouts in visually impaired men and women. Journal of sport sciences, 00(0), 1-9.

Iglesias, X., Gasset, A., González, C., \& Anguera, M. T. (2010). Interacción competitiva y presión ambiental en deportes de combate: aplicación de la metodología observacional. Revista de iberoamericana de psicología del ejercicio y del deporte, 5, 267-282.

Lapresa, D., Arana, J., Anguera, M. T., \& Garzón, B. (2013). Compartive analysis of sequentiality using SDIS-GSEQ and THEME: A concrete example in soccer. Journal of sports sciences, 31(15), 1687-1695. doi:10.1080/02640414.2013.796061.

López, C. E. \& Santoyo, V. C. (2004). Asimetría de la interacción conflictiva de cónyuges violentos: la prueba de un modelo. Revista mexicana de análisis de la conducta, 30, 115-138.

Magnusson, M. S. (2000). Discovering hidden time patterns in behavior: T-patterns and their detection. Behavior Research Methods, 32(1), 93-110.

Pedroza, C. F. J., \& Cervantes, H. A. R. (2013). Desarrollo de una herramienta observacional para recolección y análisis de comportamiento en combates de Taekwon-do. Manuscrito presentado para su publicación.

Savage, T. N., \& Stuart, M. A. (2016). Tackling reliability and construct validity: the systematic development of a qualitative protocol for skill and incident analysis. Journal of sports sciences, 21(18). doi:10.1080/02640414.2016.1172722. 